\title{
Leveling Response Factors in the Electrospray Ionization Process Using a Heated Capillary Interface
}

\author{
Jennifer L. Frahm* and David C. Muddiman* \\ Department of Biochemistry and Molecular Biology, Mayo Clinic College of Medicine, \\ Rochester, Minnesota, USA
}

Michael J. Burke

Division of Engineering, Mayo Clinic, Rochester, Minnesota, USA

Several investigators have observed a discrepancy in electrospray response of complementary strands from denatured DNA, which has been attributed to the difference in hydrophobicity between the two strands; the more hydrophobic species tend to have higher ion abundances. The implementation of a heated electrospray source has allowed us to "level" the electrospray response for two equimolar complementary strands with different hydrophobicities. As the temperature was increased, the ratio of ion abundances of the less hydrophobic noncoding strand to the more hydrophobic coding strand approached unity. Furthermore, the heated electrospray source was used to denature amplicons containing 7-deaza purines, which can be used to facilitate sequencing by mass spectrometry. (J Am Soc Mass Spectrom 2005, 16, 772-778) @ 2005 American Society for Mass Spectrometry

$\mathrm{E}$ lectrospray ionization (ESI) has become indispensable in the analysis of large biomolecules. Although ESI mechanisms are not completely characterized, investigators have recognized numerous factors that influence ESI response. One of these factors is the analyte's hydrophobicity. The correlation between ESI response and hydrophobicity of the analyte has been the subject of several investigations [1-5].

The effect an analyte's hydrophobicity on ESI response can be quantitatively estimated using an Arrhenius-type rate equation described by Fenn [1]:

$$
N_{i z}=3 A e^{\left(-\Delta G_{i z}^{o} / R T\right)}\left(N_{i} / r\right) e^{\left(\Delta r^{*} z Q / 4 \pi \varepsilon_{0} R T r^{2}\right)}
$$

Eq1 estimates the ion flux from the droplet surface, $N_{i z}$ of an ion $i$ with $z$ charges. Ion flux is a function of a constant $(A)$ that relates bulk concentration to the surface density, the free energy of solvation $\left(\Delta G_{i z}^{o}\right)$, gas constant $(R)$, temperature $(T)$, number of moles $\left(N_{i}\right)$, droplet radius $(r)$, distance ion has to travel to be free of the droplet $(\Delta r)$, number of excess charges $(Q)$, and the gas permittivity constant $\left(\varepsilon_{0}\right)$. Eq 1 is explained in detail in reference [1].

Eq 1 can be used to predict ESI response of various

Published online March 10, 2005

Address reprint requests to Dr. D. C. Muddiman, Department of Biochemistry and Molecular Biology, Mayo Clinic College of Medicine, Medical Science Building 3-115, 200 First Street SW, Rochester, MN 55905, USA. E-mail: muddiman.david@mayo.edu

* Also at the W. M. Keck FT-ICR Mass Spectrometry Laboratory, Mayo Proteomics Research Center. oligonucleotides, as described by Null et al. [5]. Briefly, eq 1 was extended to predict the ESI response for oligonucleotides based on their base composition [5]. A detailed explanation of how the values were obtained, including the assumptions made for this model, can be found in reference [5]; a correction to the values reported for the ion flux and the ion flux scores is presented here. The ion flux values should be $2.1 \times$ $10^{-28}, 2.4 \times 10^{-33}, 2.4 \times 10^{-36}$, and $1.2 \times 10^{-28} \mathrm{~mol}$ for A, C, G, and T, respectively, desorbing from the first offspring droplet at $75 \%$ of the Rayleigh limit. The negative $\log$ of the ion flux values is then used to determine the ion flux score, shown in eq 2. Eq 2 is the corrected version of eq 3 reported in reference [5], which has been amended to reflect the ion flux corrections. An ion flux score is an estimation of the ion flux (eq 1) for an oligonucleotide calculated when the first offspring droplet reaches $75 \%$ of the Rayleigh limit during the electrospray process normalized to the length [5].

$$
\begin{aligned}
& \text { ion flux score }= \frac{\sum-\log \left(N_{i z}\right)}{L} \\
&=\frac{(\# A \times 27.7)+(\# C \times 32.6)}{+(\# G \times 35.6)+(\# T \times 27.9)} \\
& L
\end{aligned}
$$

Species with higher ion flux scores will be less abundant in the mass spectrum. Ion flux scores suggest a semiquantitative estimation of ion abundances that can be 
used to compare oligonucleotides. However, owing to the many assumptions required to develop eq 2 and the dynamic nature of the electrospray process, any quantitative evaluation, albeit a crude approximation, is difficult to make. Therefore, Null et al. also reported a correlation between the number of guanines in an oligonucleotide and ESI response [5]. Guanine's lower hydrophobicity and more negative free energy of solvation relative to the other nucleobases result in its lower ion abundance. The variables $A$, related to surface activity, and $\Delta G_{i z}^{o}$, the amount of work necessary to desorb the ion from the droplet, are characteristic to the species in solution [5]. The remaining variables will be constant for a given droplet.

An increase in the hydrophobicity of an analyte will increases its ion abundance in the mass spectrum. It has been previously reported that the addition of an alkyl chain (hydrophobic tail) to the $5^{\prime}$ terminus selectively enhances the signal of one strand over the complementary strand from denatured amplicons [5]. The addition of an alkyl chain increases the hydrophobicity of the molecule, consequently promoting its escape from the droplet [5]. As a molecule becomes more hydrophobic, the surface activity (encompassed in constant $A$ in eq 1 ) leads to a lower ion flux score (i.e., higher ion abundance).

Improvements in ion abundance have also been observed when the electrospray solution is heated [6]. Ikonomou and Kebarle describe two rationales for the increase in ion abundance of several species studied in water when heat is applied; (1) better solvent evaporation, which thereby aids analyte fission from the droplet and (2) reduction of water surface tension as the temperature increases, which reduces the electric field required for the onset of electrospray and thus reduces the adverse effects of corona discharge [6].

Several investigators have reported the use of a heated electrospray interface for a variety of applications [6-11]. Originally, our laboratory designed a heated electrospray source in an attempt to denature duplex DNA [7]. Herein, we describe a completely redesigned source that is more stable, allowing for broader applications. As the temperature of the ESI solution was increased, we observed a leveling effect on the ion abundance of two complementary strands of denatured DNA with different hydrophobicities. Also, we demonstrate that the heated electrospray source denatures duplex DNA including amplicons containing the more stable 7-deaza purines. The utility of 7-deaza purines for sequencing analysis has been previously reported [13, 14]. In 7-deaza analogs, the N7 nitrogen is replaced with carbon, eliminating the preferred protonation site, which makes the site resistant to fragmentation [12]. However, the effect of the carbon substitution on the duplex stability is less clear. Grein et al. report that the duplex stabilization by 7-deaza purines may depend on the sequence and the salt conditions [15]. Our laboratory has observed that denaturation of oligonucleotides containing deaza analogs is more dif- ficult compared to the unmodied oligonucleotides (unpublished observations).

\section{Experimental}

\section{PCR Amplification}

A 53-base pair region containing a single nucleotide polymorphism (SNP) (dbSNP, rs no.717336) was amplified from synthetic template designed from a sequence on the long arm of chromosome 22 (SNP 22). Each PCR reaction contained 1X AmpliTaq Gold PCR buffer, 3 $\mathrm{mM} \mathrm{MgCl} 2,0.2 \mathrm{mM}$ each deoxynucleotide triphosphate, 12.5 pmol of each primer (forward sequence, 5' GGG GCC ATC AGA GAA ATA TAC CT 3', reverse sequence, 5' CCA GGG TGG TAA TTT CCA GGT 3') (Midland Certified Reagent Co., Midland, TX), 1 fmol template (Midland Reagent Co., Midland, TX), and 1.25 U of AmpliTaq Gold (Applied Biosystems, Foster City, CA) in a total volume of $50 \mu \mathrm{L}$. Amplification was performed in a 96-well MJ Research PTC 200 DNA Engine System Peltier Thermal Cycler (Watertown, MA) using a hot-start PCR program. Cycling conditions were as follows: an initial ten-min heat step at $95^{\circ} \mathrm{C}$ (required for enzyme activation), followed by 34 cycles at $95{ }^{\circ} \mathrm{C}$ for $30 \mathrm{~s}$ (denaturation), $58^{\circ} \mathrm{C}$ for $45 \mathrm{~s}$ (annealing), and $72{ }^{\circ} \mathrm{C}$ for $45 \mathrm{~s}$ (elongation). A final elongation step was accomplished at $72{ }^{\circ} \mathrm{C}$ for $10 \mathrm{~min}$. PCR reactions containing 7-deaza analogs were prepared in accordance with the above, except that 7-deaza-2'deoxy-GTP (Roche Diagnostics Corp., Indianapolis, IN) and 7-deaza-2'-deoxy-ATP (TriLink BioTechnologies, San Diego, CA) were substituted for deoxyguanosine triphosphate and deoxyadenosine triphosphate, respectively, in separate reactions with a final concentration of $400 \mu \mathrm{M}$ per reaction. The resulting amplicons were purified before ESI-Fourier transform-ion cyclotron resonance (FT-ICR) mass spectrometry analysis according to standard practice consisting of ethanol precipitation $[16,17]$ and microdialysis [18, 19].

\section{Mass Spectrometry}

Mass spectra were acquired in negative-ion mode on a modified ESI-FT-ICR mass spectrometer (IonSpec Inc., Irvine, CA) with a 7 tesla superconducting magnet (Cryomagnetics, Oak Ridge, TN). PCR products were electrosprayed from a $15 \mu \mathrm{m}$ i.d. PicoTip Emitter (New Objective, Woburn, MA) and remotely coupled to a potential of $-2000 \mathrm{~V}$. PCR products were electrosprayed from a buffer consisting of 60:20:20 acetonitrile/isopropanol/10 $\mathrm{mM}$ ammonium acetate in water with a final concentration of $20 \mathrm{mM}$ each piperidine and imidazole and infused at a rate of $5 \mathrm{~nL} \mathrm{~s}^{-1}$. Singleacquisition spectra were collected with $1024 \mathrm{k}$ data points with a digitization rate of $1 \mathrm{MHz}$ and a Hann window function applied, then zero-filled three times prior to fast-Fourier transform. Spectra were externally calibrated using polyethylene glycol (PEG-1000). 


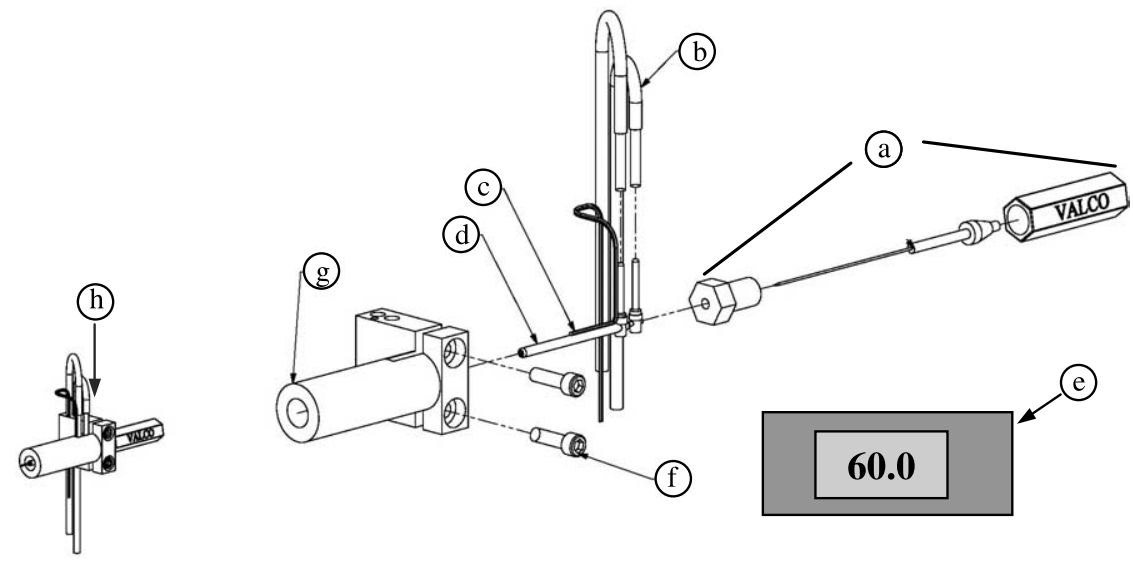

\begin{tabular}{|c|l|}
\hline Part & Name \\
\hline a & PicoTip $^{\mathrm{TM}}$ Emitter \\
\hline b & Heater Lead \\
\hline c & Thermocouple \\
\hline d & Capillary-nozzle heater assembly \\
\hline e & Controller display \\
\hline f & Hex screws \\
\hline g & Heater insulator \\
\hline h & Assembled heated ESI interface \\
\hline
\end{tabular}

Figure 1. Illustration of the heated electrospray source. (a) PicoTip Emitter; (b) heater lead; (c) thermocouple; (d); capillary-nozzle heater assembly (concentric hypodermic needles); (e) controller display; (f) Hex screws to adjust the fit of the insulator around the Valco fitting; (g) capillary-nozzle heater insulator; (h) assembled heated ESI interface.

\section{Heated Electrospray Source}

A schematic of the heated electrospray source is shown in Figure 1. The design allows for the heater and the electrospray transfer line (Figure 1a) to remain separate entities, thus permitting easy replacement of the electrospray emitter as needed. Two electrical contact pins (Figure $1 b$ ) are located at the upstream end of the ESI emitter spray. A thermocouple (Figure 1c) is attached to two soldered concentric hypodermic needle tubes (Figure 1d). The tight fit of the ESI emitter (Figure 1a) in the inner tubing is too small to accommodate a thermocouple, so the thermocouple is attached only to the outer tubing (Figure 1c). The outer tubing temperature is monitored by a $\mathrm{K}$ type thermocouple (Figure 1c) (Omega 5SRTC-TT-K-36-36, Stamford, CT) bonded to the surface about one-third of the way from the emitter of the heater, where the temperature is near its maximum over the length of the heater. The set/measured temperature is displayed on a four-digit LED readout (Figure 1e). The thermocouple signal is fed to a closed loop controller (Omega CNi3254) (Figure 1e), which controls the current to the heater through a voltage to current converter circuit. The proportional, integral, derivative (PID) controller parameters are optimized to bring the temperature to the set point as quickly as possible and maintain the temperature within error limits $\left( \pm 0.5^{\circ} \mathrm{C}\right.$ accuracy). PID parameters are proportional $42 \%$, integral 9 resets $/ \mathrm{s}$, derivative rate $2.3 \mathrm{~s}$.

The temperature of the inner tubing is linearly related to the outer temperature. Inner and outer tubing refers to the soldered concentric hypodermic needles in Figure $1 \mathrm{~d}$. This relationship was empirically determined over the controller temperature range of 23.7$77.0^{\circ} \mathrm{C}$; the relationship determined by linear least squares regression analysis is shown in eq 3 . All further discussion of temperatures of the heated electrospray source will refer to the inside temperature.

$$
\text { inner } T=1.4(\text { outer } T)-10.4
$$

Current flows down the inner hypodermic tubing and back on the outer concentric tubing, heating both sections of tubing. Since the inner tubing has a smaller cross-sectional area and therefore a higher resistance, it becomes hotter than the outer tubing. The outer tubing is still warm enough to limit heat losses from convection and radiation from the inner tubing.

\section{Results and Discussion}

Figure 2 shows an ESI-FT-ICR mass spectrum of the completely denatured SNP 22 amplicon containing 7-deaza adenine, indicated by the presence of both the 
5' GGG GCC ATC AGA GAA ATA TAC CTC TTA TTA CCA CCT GGA AAT TAC CAC CCT GG 3'

3' CCC CGG TAG TCT CTT TAT ATG GAG AAT AAT GGT GGA CCT TTA ATG GTG GGA CC 5'

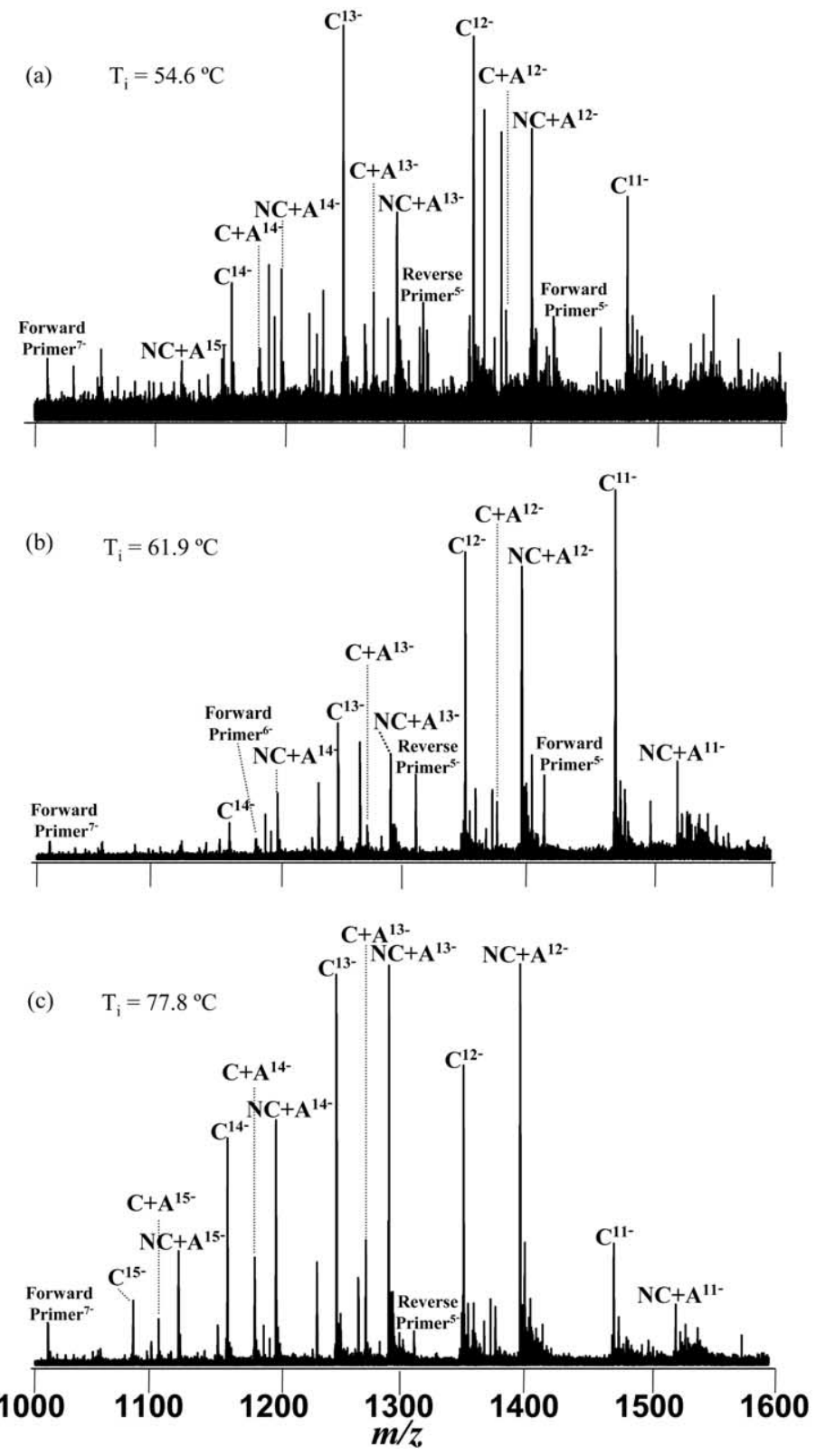

Figure 2. Single-acquisition ESI-FT-ICR mass spectra of the denatured SNP 22 amplicon containing 7-deaza adenine; sequence is shown above the spectra. Underlined portions indicate the primer sequences and 7-deaza adenines are bolded. Primer peaks are labeled accordingly. Charge states are labeled according to the strand from which the the peak originated either $\mathrm{C}$ (coding) or NC (noncoding). $\mathrm{C}+\mathrm{A}$ and $\mathrm{NC}+\mathrm{A}$ indicate the monoadenylated coding and noncoding strands, respectively. For each spectrum, the temperature inside the heater element $\left(\mathrm{T}_{\mathrm{i}}\right)$ is listed. Mass spectrum of the amplicon acquired at (a) $54.6{ }^{\circ} \mathrm{C}$; (b) $61.9^{\circ} \mathrm{C}$; (c) $77.8^{\circ} \mathrm{C}$.

coding and noncoding strands and the absence of the double stranded SNP 22 amplicon. In Figure 2, the monoadenylated noncoding strand is the sole species observed for the noncoding strand. Adenylation is an artifact of the Taq polymerase, which preferentially adenylates the $3^{\prime}$ end during PCR amplification.

The heated electrospray source also denatured the unmodified SNP 22 and SNP 22 containing 7-deaza guanine (data not shown). The ability of the newly fashioned heated electrospray source to denature amplicons containing 7-deaza analogs is a salient result, especially since the previous heated electrospray source [7] was unable to denature these species (unpublished observations).

We believe that the heater element heats the electrospray transfer line more efficiently and evenly, primar- 


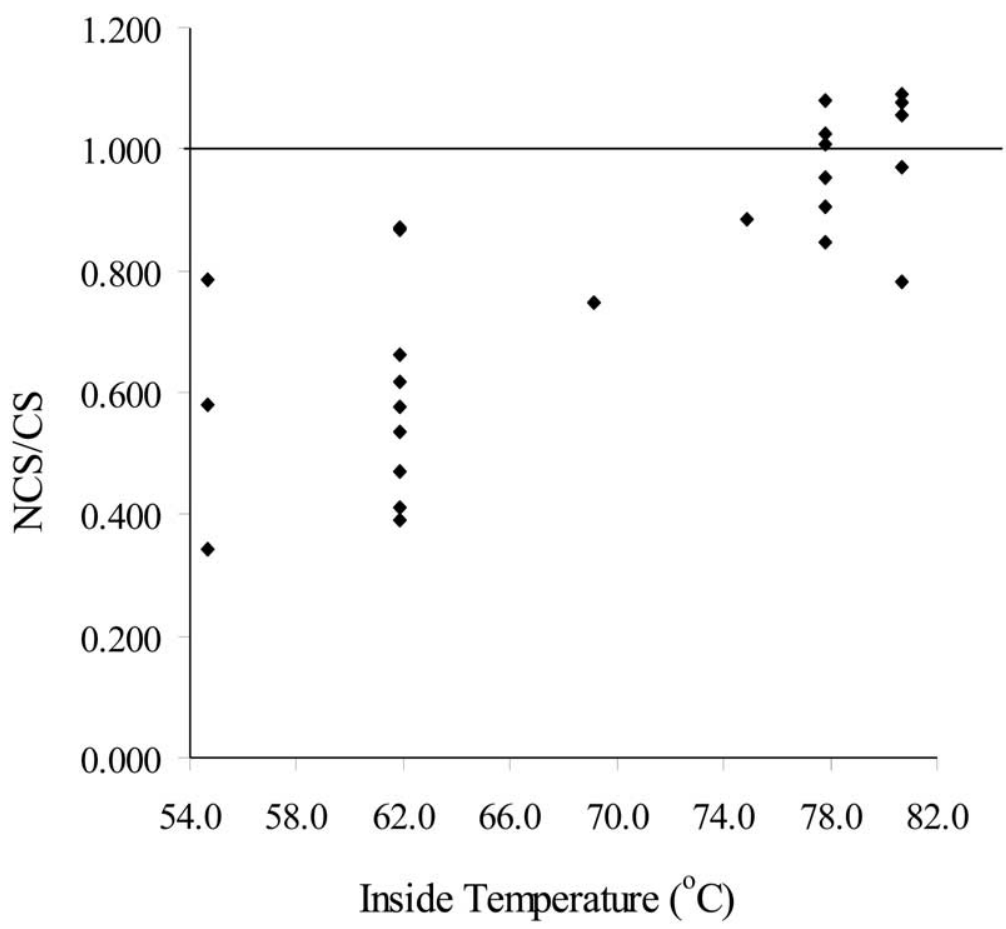

Figure 3. Plot of the ratio of ion abundances of the noncoding strand (NCS) and coding strand (CS) versus the inside temperature of the heated ESI source. The line indicates equal ESI response for the noncoding and coding strands.

ily because of the smaller diameter of the heater. As the solution begins to heat, a gas layer begins to form on the inside perimeter of the capillary. Since this gas layer acts as an insulator, preventing even heating of the solution beneath the gas, a reduction in the diameter of the heated source thereby reduces the surface area to volume ratio providing more even heating. Furthermore, the new source was designed so that the heater element surrounds the electrospray emitter, thus allowing the solution to be heated just prior to electrospray droplet formation at the tip. In the previous design, the transfer line was heated further from the tip [7], allowing the liquid ample opportunity to cool. The consequence of solution cooling may have resulted in ineffective denaturation of oligonucleotides containing 7-deaza moieties with the previous source.

In Figure 2a, a discrepancy in the ion abundance is apparent between the coding and monoadenylated noncoding strands obtained from the denatured SNP 22 amplicon (sequence shown in Figure 2). The monoadenylated noncoding strand is underrepresented in the mass spectrum. It is important to note that the solution is inherently equimolar in both strands since the species were obtained by denaturing the duplex amplicon. The signal bias between the resulting complementary strands of this amplicon has been previously observed [5].

The signal bias can be attributed to the difference in ion flux for the two species. Ion flux for the coding and monoadenylated noncoding strands can be directly related to the base compositions, which are $\mathrm{A}_{16} \mathrm{C}_{15} \mathrm{G}_{10} \mathrm{~T}_{12}$ and $\mathrm{A}_{13} \mathrm{C}_{10} \mathrm{G}_{15} \mathrm{~T}_{16}$, respectively. The monoadenylated noncoding strand contains 15 guanines, whereas the coding strand only includes 10 guanines. A higher number of molecules of the coding strand are expected to escape the droplet based on this measure of ion flux (vide supra).

When the heated electrospray source was utilized we observed an increase in the ion abundance of the monoadenylated noncoding strand relative to the ion abundance of the coding strand, shown in Figure $2 \mathrm{~b}$ and c. Additionally, an increase in temperature resulted in an improved signal-to-noise ratio, which is apparent from a comparison of Figures 2a-c. It is important to note that every spectrum was collected under the same conditions, temperature being the only variable.

Figure 3 is a graph representing the ratio of ion abundances of the noncoding strand (NCS) to the coding strand (CS) as a function of temperature. In order to make comparisons between temperatures, the same experimental conditions were utilized for each acquisition. These conditions were not optimal for the $69.1^{\circ} \mathrm{C}$ and $74.8^{\circ} \mathrm{C}$ temperatures, therefore only one spectrum could be acquired for each of these temperatures. However, these temperatures are not included in the statistical analysis and thus do not affect the conclusions. The ratio of ion abundances approaches unity as the temperature increases, demonstrating the enhanced ion abundance of the noncoding strand (i.e., less hydrophobic strand) with respect to the ion abundance of the coding strand.

A statistically significant difference in the mean ion abundance was found between the $54.6{ }^{\circ} \mathrm{C}$ and $77.8^{\circ} \mathrm{C}$ 
$(p=0.0016), 61.9^{\circ} \mathrm{C}$ and $77.8^{\circ} \mathrm{C}(p=0.0002), 54.6^{\circ} \mathrm{C}$ and $80.7^{\circ} \mathrm{C}(p=0.0012)$, and $61.9^{\circ} \mathrm{C}$ and $80.7^{\circ} \mathrm{C}(p=$ 0.0002 ) temperatures (two-sided two-sample $t$-test, Bonferroni-corrected $\alpha$-level $=0.008)$. Comparisons of temperatures to the $69.1^{\circ} \mathrm{C}$ and $74.8^{\circ} \mathrm{C}$ groups were not included as it is not statistically valid to include groups containing only one observation.

The increase in ion flux due to the increase in temperature is correlated to the free energy of solvation. The free energy of solvation can be defined as the activation-energy barrier required for the ion to escape the droplet supplied in the form of thermal energy [1]. At higher temperatures, a larger number of ions will reach the required energy of activation. Therefore, a larger number of ions will escape the droplet, resulting in increased ion abundance at higher temperatures. Alternatively, the increase in the temperature could reduce the competition between the noncoding and coding strand ions (i.e., reduced ion suppression effect) by decreasing both the droplet size and surface tension. Further experiments are necessary to elucidate which mechanism is the dominant factor giving rise to these observations.

An increase in the average charge state was also observed at higher temperatures. The average charge state at $54.6{ }^{\circ} \mathrm{C}$ for the coding strand and noncoding strand of SNP 22 is 12 . At $74.9^{\circ} \mathrm{C}$ the average charge state of the both coding and noncoding strands increases to 13 . We actually observed two groups, based on average charge state. A lower temperature (54.6$69^{\circ} \mathrm{C}$ ) group, which had an average charge state of 12 and a higher temperature $\left(74.9-83.6^{\circ} \mathrm{C}\right)$ group, which had an average charge state of 13 . This observation can be related to a phenomenon previously described by Fenn [1]. Fenn explains that a larger proportion of ions at higher charge state are formed at fast evaporation rates [1]. The rate of evaporation is increased when the ESI solution is heated, thus increasing the surfacecharge density of the droplet. Higher surface-charge densities of the droplet increase the probability that the ion will be in contact with more charges, which the ion will take upon desorption, thus leading to higher charge states [1]. More hydrophobic species dislike the solvent environment and want to escape the droplet earlier in the desolvation process when the surfacecharge density is lower, resulting in lower charge states $[1,5]$. As the temperature, and therefore, the evaporation rate increases, a higher number of ions of the less hydrophobic species desolvate from the droplet.

A potential application of the heated source is the determination of allelic frequencies from pooled DNA. It has been shown that allelic frequencies down to $1 \%$ of pooled DNA can be determined using primers with hydrophobic tails during PCR amplification [5]. Utilizing higher temperatures with the heated electrospray source to increase ion abundance, in addition to the use of primers with hydrophobic tails, the potential to detect species below the $1 \%$ level exists, which would be important for mutation sequence variations in the population that exist with a frequency of less than $1 \%$ of the population.

\section{Conclusions}

The heated source provides a simple means to obtain single-stranded species of DNA. Importantly, amplicons containing the more stable 7-deaza purines, compared to the parent purines, can be fully denatured. Information from both strands is obtained and can be used to delineate base composition. Furthermore, the heated electrospray source can be used to improve the electrospray response of species with different hydrophobicities, thus increasing the total ion abundance of the underrepresented species. Usefulness of the heated source lies in its ability to aid the desolvation process, thereby increasing ion abundance and improving signal-to-noise. The utility of the heated electrospray source is not limited to DNA; use of the source may be extended to other species, such as RNA, peptides, and proteins. Some applications including online LC-MS may also benefit from a heated electrospray interface.

\section{Acknowledgments}

The authors gratefully acknowledge financial support received from the National Institutes of Health (R01HG02159), W.M. Keck Foundation, and Mayo Clinic College of Medicine. The authors would also like to thank the reviewers for their critical review of the manuscript and insightful suggestions.

\section{References}

1. Fenn, J. B. Ion Formation from Charged Droplets-Roles of Geometry, Energy, and Time. J. Am. Soc. Mass Spectrom. 1993, $4,524-535$.

2. Cech, N. B.; Krone, J. R.; Enke, C. G. Predicting Electrospray Response from Chromatographic Retention Time. Anal. Chem. 2001, 73, 208-213.

3. Zhan, D.; Fenn, J. B. Gas Phase Hydration of Electrospray Ions from Small Peptides. Int. J. Mass Spectrom. 2002, 219, 1-10.

4. Gordon, E. F.; Mansoori, B. A.; Carroll, C. F.; Muddiman, D. C. Hydropathic Influences on the Quantification of Equine Heart Cytochrome $c$ Using Relative Ion Abundance Measurements by Electrospray Ionization Fourier Transform Ion Cyclotron Resonance Mass Spectrometry. J. Mass Spectrom. 1999, 34, 1055-1062.

5. Null, A. P.; Nepomuceno, A. I.; Muddiman, D. C. Implications of Hydrophobicity and Free Energy of Solvation for Characterization of Nucleic Acids by Electrospray Ionization Mass Spectrometry. Anal. Chem. 2003, 75, 1331-1339.

6. Ikonomou, M. G.; Kebarle, P. A Heated Electrospray Source for Mass Spectrometry of Analytes from Aqueous Solutions. J. Am. Soc. Mass Spectrom. 1994, 5, 791-799.

7. Mangrum, J. B.; Flora, J. W.; Muddiman, D. C. Solution Composition and Thermal Denaturation for the Production of Single-Stranded PCR Amplicons: Piperidine-Induced Destabilization of the DNA Duplex. J. Am. Soc. Mass Spectrom. 2002, 13, 232-240.

8. Mirza, U. A.; Cohen, S. L.; Chait, B. Heat-Induced Conformational Changes in Proteins Studied by Electrospray Ionization Mass Spectrometry. Anal. Chem. 1993, 65, 1-6. 
9. Daneshfar, R.; Kitova, E. N.; Klassen, J. S. Determination of Protein-Ligand Asssociation Thermochemistry Using Variable-Temperature Nanoelectrospray Mass Spectrometry. J. Am. Chem. Soc. 2004, 126, 4786-4787.

10. Benesch, J. L. P.; Sobott, F.; Robinson, C. V. Thermal Dissociation of Multimeric Protein Complexes by Using Nanoelectrospray Mass Spectrometry. Anal. Chem. 2003, 75, 2208-2214.

11. Rychlovsky, P.; Cernoch, P.; Sklenickova, M. Application of a Heated Electrospray Interface for On-Line Connection of the AAS Detector with HPLC for Detection of Organotin and Organolead Compounds. Anal. Bioanal. Chem. 2002, 374, 955962.

12. Kirpekar, F.; Nordhoff, E.; Kristiansen, K.; Roepstorff, P.; Hahner, S.; Hillenkamp, F. 7-Deaza Purine Bases Offer a Higher Ion Stability in the Analysis of DNA by MatrixAssisted Laser Desorption Ionization Mass Spectrometry. Rapid Commun. Mass Spectrom. 1995, 9, 525-531.

13. Hannis, J. C.; Muddiman, D. C. Tailoring the Gas-Phase Dissociation and Determining the Relative Energy of Activation for Dissociation of 7-Deaza Purine Modified Oligonucleotides Containing a Repeating Motif. Int. J. Mass Spectrom. 2002, 219, 139-150.
14. Frahm, J. L.; Mason, C.; Muddiman, D. C. Utility of Accurate Monoisotopic Mass Measurements to Confidently Identify Lambda Exonuclease Generated Single-Stranded Amplicons Containing 7-Deaza Analogs by Electrospray Ionization FTICR Mass Spectrometry. Int. J. Mass Spectrom. 2004, 234, 79-87.

15. Grein, T.; Lampe, S.; Mersmann, K.; Rosemeyer, H.; Thomas; Seela, F. 3-Deaza and 7-Deaza purines: Duplex Stability of Oligonucleotides Containing Modified Adenine or Guanine Bases. Bioorg. Med. Chem. Lett. 1994, 4, 971-976.

16. Stults, J. T.; Marsters, J. C. Improved Electrospray Ionization of Synthetic Oligodeoxynucleotides. Rapid Commun. Mass Spectrom. 1991, 5, 359-363.

17. Limbach, P. A.; Crain, P. F.; McCloskey, J. A. Molecular Mass Measurement of Intact Ribonucleic Acids via Electrospray Ionization Quadrupole Mass Spectrometry. J. Am. Soc. Mass Spectrom. 1995, 6, 27-39.

18. Liu, C.; Muddiman, D. C.; Smith, R. D. Improving the Microdialysis Procedure for Electrospray Ionization Mass Spectrometry of Biological Samples. J. Mass Spectrom. 1997, 32, 425-431.

19. Hannis, J. C.; Muddiman, D. C. Characterization of a Microdialysis Approach for the Preparation of PCR Products for ESI-MS Using ICP-AES and On-Line UV-Vis Detection. Rapid Commun. Mass Spectrom. 1999, 13, 323-330. 